\title{
Tuberculosis pulmonar presentada como neumotórax masivo
}

\author{
Pulmonary tuberculosis disguised as a massive pneumothorax
}

\author{
Marta Rebocho Alves ${ }^{1}$, Tânia Araújo Ferreira ${ }^{1}$ \\ ${ }^{1}$ Centro Hospitalar Universitário do Porto
}

\begin{abstract}
Pulmonary tuberculosis is an indolent disease that may rarely be fatal. Extensive destruction of pulmonary parenchyma is rarely seen nowadays. This image shows a large tuberculosis cavitation that mimics massive a pneumothorax.

Keywords: tuberculosis, pneumothorax, chest tubes, neoplasms, emergency medicine.

Palabras clave: tuberculosis, neumotórax, tubo torácico, neoplasia, medicina de emergencias.
\end{abstract}

A 47-year-old women was admitted to the emergency department with asthenia, anorexia, weight loss, night sweats, purulent cough for several months, and aggravating dyspnoea and chest pain in the previous week. Five years before she was advised to undergo thyroidectomy due to a suspicious nodule which she refused and abandoned all follow-up. She presented extremely undernourished, with severe hypoxemic respiratory failure but was otherwise stable. Initial chest X-ray (figure 1) showed a left lung infiltrate and an apparent total right lung pneumothorax. Due to haemodynamic stability pneumothorax was not immediately approached and was better accessed with a CT-scan (figure 2). It showed a right large pneumothorax (yellow arrow) with slight mediastinal deviation; large cavitation in almost all superior right lobe (white arrow) with thick walls, gas and liquid inside (green arrow); smaller lower lobe cavitation, one of which in continuity with the pneumothorax; collapse of the right lung; left lung with tree-in-bud pattern consolidations (orange arrows); and a large suspicious nodule with contrast enhancement on the thyroid gland. These findings, associated with the patient's complaints, rose the suspicion of pulmonary tuberculosis with cavitation, and a chest tube was not placed given the aetiology of the pneumothorax. Despite organ support and anti-tuberculosis drugs (isoniazid, rifampicin, pyrazinamide and ethambutol), she developed septic shock, refractory respiratory failure and died within less than 48 hours of treatment. She was HIV negative and had normal HbA1C. Koch bacillus was isolated on sputum posthumously (Lowenstein culture). Whereas active pulmonary tuberculosis and disseminated tuberculosis are associated with HIV seropositivity and immunosuppressive therapy, ${ }^{1,2}$ cavitation is mainly associated with diabetes, old-age and cancer, mostly haematological and head/neck cancer, as in this case. ${ }^{3,4}$ Furthermore, tuberculosis associated pneumothorax has a worse prognosis. ${ }^{5}$ We emphasize the importance of clarifying the aetiology of a pneumothorax, since its management may depend on it, and mainly when its apparent extent does not fit the clinical picture. In this case a chest tube based on the initial Xray image could have aggravated her status by inducing upper lobe cavitation rupture and worsening the pre-existent pneumothorax.

\section{CONFLICTO DE INTERESES}

No existen conflictos de interés por parte de los autores de este trabajo.

\section{FINANCIACIÓN}

No ha existido financiación externa de ningún tipo para llevar a cabo el presente trabajo.

\section{ASPECTOS ÉTICOS}

Se ha solicitado consentimiento informado explíito a los pacientes participantes en el presente trabajo.
Figure 1. Postero-anterior chest $X$-ray.

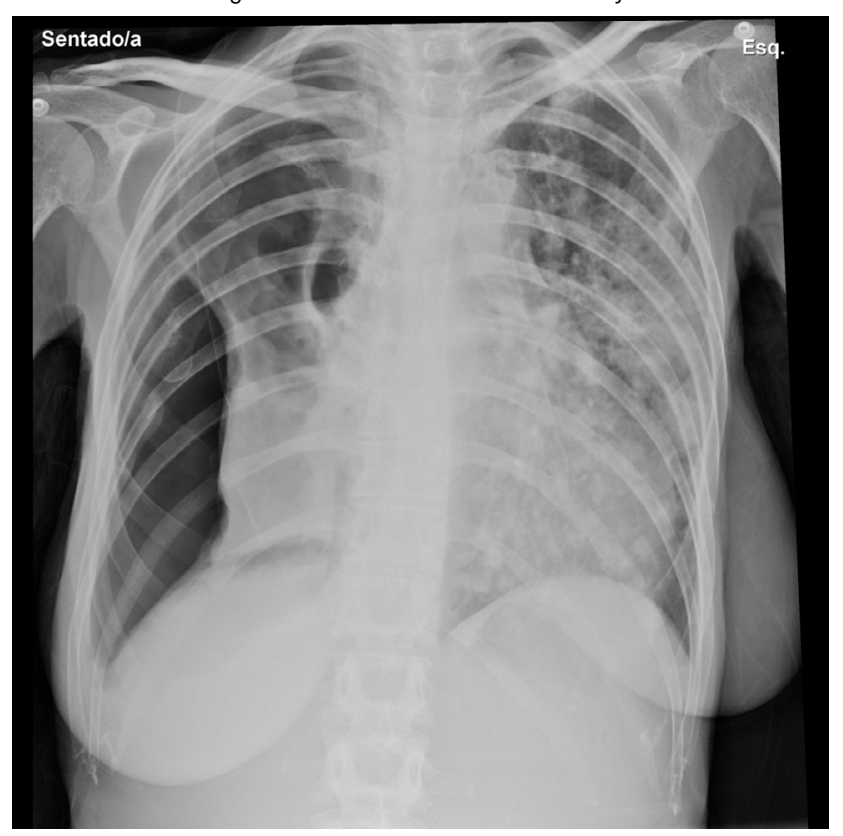

Figure 2. CT scan coronal and axial views.

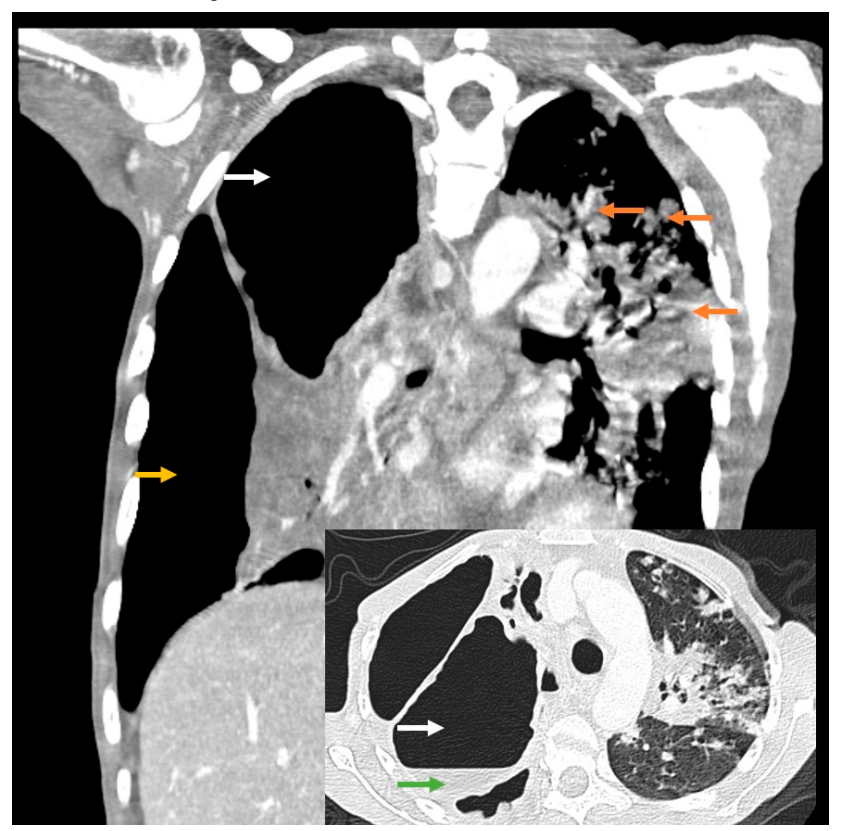




\section{BIBLIOGRAFÍA}

1. Bell LCK, Noursadeghi M. Pathogenesis of HIV-1 and Mycobacterium tuberculosis co-infection. Nat Rev Microbiol. 2018;16(2):80-90. doi:10.1038/nrmicro.2017.128

2. Alende-Castro V, Macía-Rodríguez C, Páez-Guillán E, García-Villafranca A. Miliary pattern, a classic pulmonary finding of tuberculosis disease. Journal of Clinical Tuberculosis and Other Mycobacterial Diseases. 2020;20:100179. doi:10.1016/j. jctube.2020.100179

3. Urbanowski ME, Ordonez AA, Ruiz-Bedoya CA, Jain SK, Bishai WR. Cavitary tuberculosis: the gateway of disease transmission. The Lancet Infectious Diseases. 2020;20(6):e117-e128. doi:10.1016/S1473-3099(20)30148-1

4. Kamboj M, Sepkowitz KA. The risk of tuberculosis in patients with cancer. Clin Infect Dis. 2006;42(11):1592-1595. doi:10.1086/503917

5. Shamaei M, Tabarsi P, Pojhan S, et al. Tuberculosis-Associated Secondary Pneumothorax: A Retrospective Study of 53 Patients. Respiratory Care. 2011;56(3):298-302. doi:10.4187/respcare.00695 